\title{
Early detection of diseases in plant tissue using spectroscopy - applications and limitations
}

\begin{abstract}
Plant diseases can greatly affect the total production of food and agricultural materials, which may lead to high amount of losses in terms of quality, quantity and also in economic sense. To reduce the losses due to plant diseases, early diseases detection either based on a visual inspection or laboratory tests are widely employed. However, these techniques are laborintensive and time consuming. In a view to overcome the shortcoming of these conventional approaches, several researchers have developed non-invasive techniques. Recently, spectroscopy technique has become one of the most available non-invasive methods utilized in detecting plant diseases. However, most of the studies on the application of this novel technology are still in the experimental stages, and are carried out in isolation with no comprehensive information on the most suitable approach. This problem could affect the advancement and commercialization of spectroscopy technology in early plant disease detection. Here, we review the applications and limitations of spectroscopy techniques (visible/infrared, electrical impedance and fluorescence spectroscopy) in early detection of plant disease. Particular emphasis was given to different spectral level, challenges and future outlook.
\end{abstract}

Keyword: Plant diseases; Spectroscopy techniques; VIS/IR spectroscopy; Impedance spectroscopy; Fluorescence spectroscopy 